\title{
Heterogeneity of alkane chain length in freshwater and marine cyanobacteria
}

\author{
Tabinda Shakeel ${ }^{1}$, Zia Fatma ${ }^{1}$, Tasneem Fatma ${ }^{2}$ and Syed Shams Yazdani ${ }^{1,3}$ * \\ 'Synthetic Biology and Biofuels Group, International Centre for Genetic Engineering and Biotechnology, New Delhi, India \\ 2 Department of Biosciences, Jamia Millia Islamia, New Delhi, India \\ ${ }^{3}$ DBT-ICGEB Centre for Advanced Bioenergy Research, International Centre for Genetic Engineering and Biotechnology, New Delhi, India
}

Edited by:

M. Kalim Akhtar, University of

Edinburgh, UK

\section{Reviewed by:}

Weiwen Zhang, Tianjin University, China

Yumiko Sakuragi, University of

Copenhagen, Denmark

${ }^{*}$ Correspondence:

Syed Shams Yazdani, Synthetic Biology and Biofuels Group,

International Centre for Genetic Engineering and Biotechnology, Aruna Asaf Ali Marg, New Delhi 110067, India

e-mail:shams@icgeb.res.in
The potential utilization of cyanobacteria for the biological production of alkanes represents an exceptional system for the next generation of biofuels. Here, we analyzed a diverse group of freshwater and marine cyanobacterial isolates from Indian culture collections for their ability to produce both alkanes and alkenes. Among the 50 cyanobacterial isolates screened, 32 isolates; 14 freshwater and 18 marine isolates; produced predominantly alkanes. The GC-MS/MS profiles revealed a higher percentage of pentadecane and heptadecane production for marine and freshwater strains, respectively. Oscillatoria species were found to be the highest producers of alkanes. Among the freshwater isolates, Oscillatoria CCC305 produced the maximum alkane level with $0.43 \mu \mathrm{g} / \mathrm{mg}$ dry cell weight, while Oscillatoria formosa BDU30603 was the highest producer among the marine isolates with $0.13 \mu \mathrm{g} / \mathrm{mg}$ dry cell weight. Culturing these strains under different media compositions showed that the alkane chain length was not influenced by the growth medium but was rather an inherent property of the strains. Analysis of the cellular fatty acid content indicated the presence of predominantly $\mathrm{C} 16$ chain length fatty acids in marine strains, while the proportion of $\mathrm{C} 18$ chain length fatty acids increased in the majority of freshwater strains. These results correlated with alkane chain length specificity of marine and freshwater isolates indicating that alkane chain lengths may be primarily determined by the fatty acid synthesis pathway. Moreover, the phylogenetic analysis showed clustering of pentadecane-producing marine strains that was distinct from heptadecane-producing freshwater strains strongly suggesting a close association between alkane chain length and the cyanobacteria habitat.

Keywords: cyanobacteria, marine strain, freshwater strain, hydrocarbon profile, fatty acid synthesis pathway, phylogenetic analysis

\section{INTRODUCTION}

The increased carbon footprint and the rapidly depleting petroleum reserves have necessitated the development of responsible, environment-friendly alternatives with immediate impact (Timilsina, 2013). Within this context, the biological production of alkanes, which can serve as drop-in fuels, offers a possible solution. As major constituents of diesel, gasoline, and jet fuels along with their high-energy content and compatibility with the existing fossil fuel infrastructure, alkanes are ideal candidates in this regard (Speight, 1997).

Several prokaryotic and eukaryotic organisms that naturally produce hydrocarbons have been reported in the literature (Coates et al., 2014). The most prominent among these are the cyanobacteria (Winters et al., 1969), which produce both alkanes and alkenes using fatty acid precursors via two different pathways (Schirmer et al., 2010; Mendez-Perez et al., 2011). One pathway involves two terminal enzymes, acyl-acyl carrier protein reductase (AAR) and aldehyde deformylating oxygenase (ADO), which successively convert fatty acyl intermediates to alkanes (Schirmer et al., 2010; Li et al., 2011, 2012; Zhang et al., 2013). Through another pathway, medium chain alkenes (also known as $\alpha$-olefins) are produced via large multi-domain protein homologous to type I polyketide synthases (PKS). Here, the fatty acyl-ACP is converted to an $\alpha$-olefin via elongation-decarboxylation mechanism leading to odd-numbered carbon chain with the double bond at the $\alpha$ position (Mendez-Perez et al., 2011). There have been no cyanobacterial species described so far with genomes that harbor both these pathways (Coates et al., 2014). However, it appears from the recent study that the AAR/ADO pathway is prevalent in alkaneproducing cyanobacteria (Klähn et al., 2014). In an earlier study, deletion of these two genes rendered cyanobacteria incapable of producing alkanes with no obvious impact on the growth of these cyanobacteria (Schirmer et al., 2010). This suggested that alkane production is not imperative for the survival of these cyanobacteria. However, this observation also raised the question about the relevance of alkane production in cyanobacteria since even after considerable transcriptomic analysis of alkane-producing cyanobacteria under various growth conditions, there was no clear correlation between AAR/ADO expression and the environment (Hernandez-Prieto and Futschik, 2012; Klähn et al., 2014). Moreover, since different chain length alkanes were observed in different cyanobacteria, it is difficult to predict whether the difference in 
chain length was due to the genetic factors or the diverse climatic condition in which they are naturally found (Schirmer et al., 2010).

Cyanobacteria are found in a variety of habitats (Cameron, 1962; Miller and Castenholz, 2000; Garcia-Pichel et al., 2001), with the most prominent ones being found in marine and freshwater environment (Reed et al., 1984; Nadeau and Castenholz, 2000; Martins et al., 2011). Several useful compounds from marine cyanobacteria and its commercial potential have been well documented (Bloch and Tardieu-Guigues, 2014). However, no systematic study has been reported comparing the alkane and/or alkene production capabilities of marine and freshwater cyanobacterial strains.

In this study, we selected cyanobacterial isolates from Indian marine and freshwater habitats and screened them for the production of alkanes and alkenes. We found that carbon chain lengths correlated well with the natural habitat of that cyanobacterium but not with the cultivation media used in the laboratory. We further compared the intrinsic ability of cyanobacteria to produce different chain length fatty acids with the alkane chain length profile. Finally, we performed a phylogenetic analysis of the $16 \mathrm{~S}$ rDNA sequences of alkane-producing strains and show a distinct clustering of marine and freshwater cyanobacteria.

\section{MATERIALS AND METHODS COLLECTION AND MAINTENANCE OF CYANOBACTERIAL CULTURES Source}

Indian freshwater cyanobacterial isolates were procured from the National Centre for Culture Collection and Utilization of Blue Green Algae, Indian Agriculture Research Institute (IARI), New Delhi and marine cyanobacterial isolates from National Facility for Marine Cyanobacteria (NFMC), Thiruchirapalli, India. Synechococcus elongatus PCC7942 and S. elongatus PCC7002 were obtained from Pasteur Culture Collection of Cyanobacteria, France.

\section{Culture medium and maintenance}

The freshwater strains were cultured in BG-11 medium while the marine isolates were cultured in ASN-III medium (Rippka et al., 1979). Experimental cultures were incubated in identical condition at light intensity $49.5 \pm 6.6 \mu \mathrm{molm}^{-2} \mathrm{~s}^{-1}$; photoperiod $16: 8 \mathrm{~h}$ (light: dark); temperature $25-30^{\circ} \mathrm{C}$ and $\mathrm{pH} 7.3 \pm 0.1$ with a salinity preference of $25 \mathrm{ppt}$ in case of marine strains.

\section{STRAIN ISOLATION}

Strain isolation was achieved via antibiotic treatment and/or dilution plating (Sarchizian and Ardelean, 2010). For antibiotic treatment, the cultures $(20 \mathrm{~mL}$ each $)$ were incubated for $1 \mathrm{~h}$ at $25-30^{\circ} \mathrm{C}$ in BG-11 or ASN-III medium containing $0.1 \times$ LB (tryptone $1 \mathrm{~g} / \mathrm{L}$, yeast extract $-0.5 \mathrm{~g} / \mathrm{L}, \mathrm{NaCl}-1 \mathrm{~g} / \mathrm{L})$, followed by the addition of antibiotics - penicillin $\mathrm{G}(100 \mu \mathrm{g} / \mathrm{ml})$, streptomycin $(50 \mu \mathrm{g} / \mathrm{ml})$, chloramphenicol $(10 \mu \mathrm{g} / \mathrm{ml})$, and augmentin $(100 \mu \mathrm{g} / \mathrm{ml})$ to each of the culture tubes. The samples were incubated for $24 \mathrm{~h}$ in dark, washed with sterile water and then incubated in the respective medium (without antibiotics) in light for $24 \mathrm{~h}$. For isolation via dilution plating, cyanobacterial strains were cultured in their respective medium until the log phase. A small volume of each culture was withdrawn and subjected to washing with sterile water followed by washing with respective sterilized medium. Serial dilutions of the cultures were prepared (dilutions up to 1000 times) and plated on BG-11-agar/ASN-III-agar plates. For both methods, strain purity was checked by growing the strains overnight in LB medium in dark.

\section{GROWTH CURVE OF CYANOBACTERIA}

The growth characteristics of alkane-producing cyanobacteria were analyzed in liquid culture. Equal amount of samples of freshwater and marine cyanobacterial cultures corresponding to a final concentration of $0.02 \mathrm{mg}$ dry cell weight $/ \mathrm{mL}$ were inoculated in their respective media in $50 \mathrm{~mL}$ volume for each time point and grown for 10 days under optimal growth conditions as mentioned in the previous section. Each $50 \mathrm{~mL}$ culture was harvested after an interval of $24 \mathrm{~h}$ (up to the tenth day after inoculation) and the samples were centrifuged and dried in the oven at $65^{\circ} \mathrm{C}$. Multiple readings were taken during the incubation period until a constant weight was attained by all the samples (Figure S1 in Supplementary Material). The dry weight of each of the samples was measured and normalized per milliliter of the culture. Strain cultivation was performed in triplicate and data plotted as an average. The hydrocarbon estimation was performed for the cultures harvested from day 5 to 9 .

\section{DETERMINATION OF THE ALKANE AND ALKENE CONTENT}

For alkane and alkene analysis, the strains were grown in $1 \mathrm{~L}$ culture in Erlenmeyer flasks at $25-30^{\circ} \mathrm{C}$ for 8 days and the hydrocarbons were extracted and analyzed using the protocol modified from Schirmer et al. (2010). Briefly, the sample volume corresponding to $20 \mathrm{mg}$ dry cell weight [cells dried at $65^{\circ} \mathrm{C}$ until the weight was constant (Figure S1 in Supplementary Material)] was withdrawn from the culture, centrifuged for $1 \mathrm{~min}$ at 13,000 rpm, resuspended in methanol and sonicated using a Sonics VibraCell for $10 \mathrm{~min}$ at amplitude of $30 \%$ and a pulse of $9.9 \mathrm{~s}$ on/off. After centrifugation for $3 \mathrm{~min}$ at $13,000 \mathrm{rpm}$, the supernatants were transferred to fresh vials and analyzed on a $7890 \mathrm{~A}$ gas chromatography system equipped with a 7000 GC/MS triple quadrapole system (Agilent). The HP-5 capillary column ( $30 \mathrm{~m}$ length, $0.32 \mathrm{~mm}$ internal diameter, $0.25 \mu \mathrm{m}$ film thickness) was used for the separation of metabolites with following method parameters: $1 \mu \mathrm{L}$ sample was injected (splitless) onto the GC-MS column with inlet temperature at $150^{\circ} \mathrm{C}$, the oven was held at $100^{\circ} \mathrm{C}$ for $3 \mathrm{~min}$. The oven temperature was ramped up to $300^{\circ} \mathrm{C}$ at a rate of $20^{\circ} \mathrm{C} / \mathrm{min}$ and was then held at $300^{\circ} \mathrm{C}$ for an additional $5 \mathrm{~min}$. The MS quadrupole scanned from 50 to $550 \mathrm{~m} / z$. Retention times and fragmentation patterns of product peaks were compared with authentic references to confirm peak identity. The hydrocarbon concentrations were quantified through multiple reaction monitoring (MRM) mode based on commercial standards (Absolute Standards).

\section{DETERMINATION OF THE FATTY ACID CONTENT}

For the analysis of the cellular fatty acid content, cyanobacterial cultures were harvested on day 8. Cell pellets were dried in the oven at $65^{\circ} \mathrm{C}$ and then crushed using pestle and mortar (Ahmad et al., 2012). The dried sample (100 mg) was used for fatty acids extraction with chloroform and methanol and finally with hexane. Trans-esterification was done using $2 \mathrm{M}$ potassium hydroxide in 
methanol. The upper hexane layer was collected, filtered, and transferred to fresh vials for fatty acyl methyl ester (FAME) analysis by GC/MS triple quadrapole system. Quantification of FAME was carried out using the 7890 A gas chromatography system equipped with Omega Wax 250 column (30 m length, $0.25 \mathrm{~mm}$ internal diameter, $0.25 \mu \mathrm{m}$ film thickness). The following method parameters were employed: $1 \mu \mathrm{L}$ sample was injected (split ratio 1:10; inlet temperature held at $150^{\circ} \mathrm{C}$ ) onto the $\mathrm{GC}$ column, the oven was held at $140^{\circ} \mathrm{C}$ for $5 \mathrm{~min}$. The oven temperature was ramped up to $240^{\circ} \mathrm{C}$ at a rate of $4^{\circ} \mathrm{C} / \mathrm{min}$ and was then held at $240^{\circ} \mathrm{C}$ for an additional $20 \mathrm{~min}$. Supelco ${ }^{\circledR} 37$ component FAME mix (Sigma Aldrich) was used as standard to identify the retention times and fragmentation patterns of the product peaks and for quantification of the specific FAME.

\section{PHYLOGENETIC ANALYSIS}

Genomic DNA from the cyanobacterial host was isolated by the conventional hexadecyl-trimethyl-ammonium bromide (CTAB) method (Zhang et al., 2014) and was used as template to amplify the $16 \mathrm{~S}$ rDNA sequence with the help of universal primers 27F ( $5^{\prime}$-AGAGTTTGATCMTGGCTCAG $-3^{\prime}$ ) and 1492R (5'-TACGGYTACCTTGTTACGACTT- $\left.3^{\prime}\right)$. The PCR product was analyzed on a $1 \%$ agarose gel in the presence of ethidium bromide, purified using a gel extraction kit (Genetix) and sequenced at Macrogen Inc. The phylogenetic analysis of cyanobacterial strains was conducted in MEGA6 (Tamura et al., 2013) with the help of $16 \mathrm{~S}$ rDNA sequences using the Neighbor-Joining method (Saitou and Nei, 1987). The bootstrap analysis was performed employing 1000 bootstrap replications (Felsenstein, 1985). The evolutionary distances were computed using the $p$-distance method (Nei and Kumar, 2000).

\section{RESULTS \\ SCREENING OF FRESHWATER AND MARINE CYANOBACTERIA FOR ALKANE AND ALKENE PRODUCTION}

We obtained 21 freshwater and 29 marine cyanobacterial strains from IARI and NFMC culture collections, respectively (Table 1). Since most of these cultures were contaminated with bacteria, we initially attempted to isolate the cyanobacterial strains via antibiotic treatment. However, many antibiotics interfered with the growth of cyanobacteria and, in a large number of cases, did not reduce the contamination. We therefore resorted to serial dilution to isolate the cyanobacterial strains by transferring four to five times on agar plates. The purity of the cyanobacterial culture was verified by growth on LB liquid medium, LB agar plate, and bright field microscopy.

After cultivation for 8 days, alkanes and/or alkenes were extracted from both the freshwater and marine strains cultivated in their respective liquid media and analyzed via GC-MS. S. elongatus PCC7942 was used as a positive control strain and S. elongatus PCC7002 was used as a negative control strain due to the presence and absence of the alkane-producing AAR/ADO pathway, respectively (Schirmer et al., 2010). A total of 32 cyanobacterial strains, which included 14 freshwater and 18 marine strains, were found to produce significant quantities of alkanes though alkenes were also detected (Table S1 in Supplementary Material). We selected these strains and analyzed the chain lengths of the alkanes

Table 1 | List of cyanobacterial strains analyzed for alkane and alkene production.

Freshwater strains (IARI Marine strains (NFMC culture collection) culture collection)

Anabaena CCC531

Aulosira sp. CCC444

Calothrix sp. CCC236

Chrococcus CCC429

Cylindrospermum CCC251

Hapalosiphon sp. CCC51

Lyngbya sp. CCC473

Nostoc muscorum CCC92

Oscillatoria sp. CCC305

Oscillatoria sp. CCC309

Phormidium sp. CCC191

Phormidium sp. CCC236

Phormidium sp. CCC317

Phormidium sp. CCC469

Phormidium sp. CCC470

Phormidium sp. CCC495

Plectonema sp. CCC475

Spirulina platensis CCC477

Synechococcus sp. CCC436

Tolypothrix CCC443

Westiellopsis CCC4

\begin{abstract}
Aphanocapsa litorallis BDU130182
Aphanocapsa sp. BDHKU35702

Aphanothece sp. BDHKU40501

Gleocapsa crepidinum BDHKU10204

Gleocapsa gigantea BDU10011

Lyngbya confervoides BDU142001

Lyngbya sp. BDU90901
\end{abstract}

Microcoleus acutissimus BDU140572

Myxosarcina sp. BDHKU33603

Myxosarcina spectalis BDU40882

Oscillatoria boryana BDU91531

Oscillatoria formosa BDU30603

Oscillatoria jasorensis BDU51031

Oscillatoria laetivirens BDU100891

Oscillatoria minnesotensis BDU41121

Oscillatoria salina BDU10142

Oscillatoria willei BDU141541

Phormidium angustissimum BDU100401

Phormidium corium BDU30241

Phormidium fragile BDU42911

Phormidium sp. BDUN661

Phormidium tenue BDU40061

Phormidium valderianum BDU40231

Plectonema terebrans BDU141661

Pseudanabaena schmidlei BDU20761

Spirulina sp. BDU51781

Spirulina subsalsa BDU30311

Synechococcus elongatus BDU130192

Synechocystis pevaleikii BDHKU35101

produced along with their quantification through MRM mode of triple quadrapole mass spectrometer. The majority of these strains produced pentadecane and heptadecane (Figure 1). The freshwater and marine strains predominantly produced heptadecane and pentadecane, respectively, though a few marine stains also produced hexadecane. Oscillatoria strains were the highest producers of alkanes for both the freshwater and marine categories. Oscillatoria CCC305 produced a total alkane content of $0.43 \mu \mathrm{g} / \mathrm{mg}$ dry cell wt, which was three times higher than the amount produced by the best alkane-producing marine strain Oscillatoria formosa BDU30603 (0.13 $\mu \mathrm{g} / \mathrm{mg}$ dry cell wt), and more than five times higher than the amount produced by S. elongatus PCC7942 $(0.083 \mu \mathrm{g} / \mathrm{mg}$ dry cell wt) (Figure 1). The representative plots of the full GC-MS scan and the alkane/alkene quantitation of these strains through MRM are given in Figure 2 and Figure S2 in Supplementary Material, respectively. Phormidium sp. were the second highest alkane-producers for both freshwater and marine cyanobacteria. S. elongatus PCC7942, a freshwater isolate, produced $0.022 \mu \mathrm{g} / \mathrm{mg}$ dry cell wt pentadecane and $0.0076 \mu \mathrm{g} / \mathrm{mg}$ dry cell wt heptadecane. This was in stark contrast to the other 


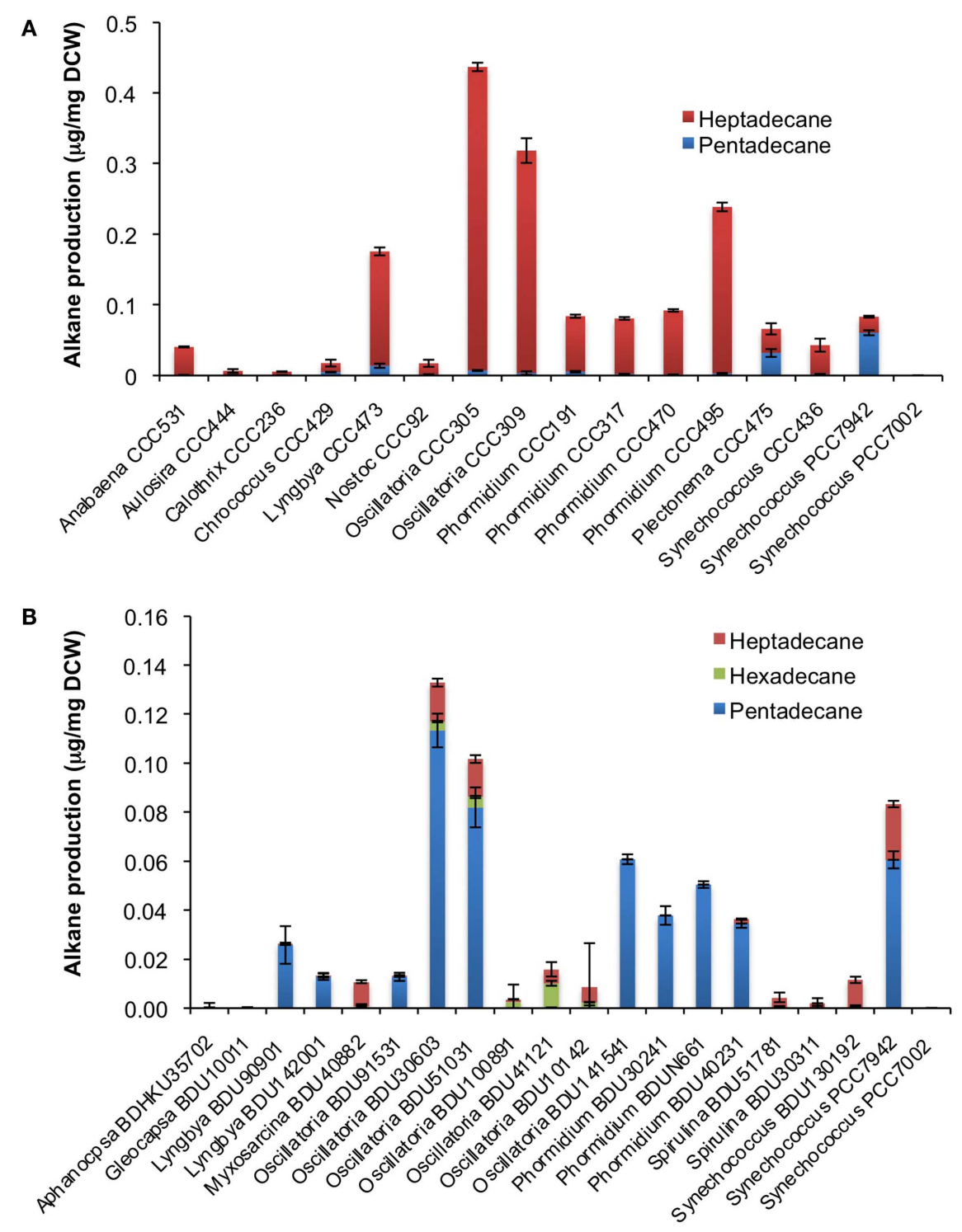

FIGURE 1 |Alkane profile of freshwater (A) and marine (B) cyanobacterial strains. Data represent average and SD of three independent experiments.

freshwater strains, which predominantly produced heptadecane. As expected, S. elongatus PCC7002, which was used as a negative control strain, did not shows any alkane and/or alkene production. Growth curves were obtained for strains, which produced significant quantities of alkane in order to identify optimum levels of alkane production (Figure 3). For most of the strains, the exponential growth phase ranged from 2 to 8 days after which point the growth rate declined. We analyzed alkane production between day 5 and 9 of growth. The trend showed an increase in alkane production until day 8 for most of the strains and then reaching saturation (Figure 4).

\section{IMPACT OF CULTIVATION MEDIA ON HYDROCARBON PRODUCTION}

Based on our GC-MS/MS analyses, we observed that the majority of freshwater cyanobacterial strains produced heptadecane as the predominant alkane while marine strains produced pentadecane. To investigate whether alkane chain length is associated with differences in composition of the cultivation media, we grew five (highest alkane-producers) of the freshwater strains in ASN-III medium, which has high-salt concentration and is generally used to grow marine strains, and five (highest alkane-producers) of the marine strains in BG-11 medium, which has low-salt concentration and is commonly used for growing freshwater strains. $S$. elongatus PCC 7942, classified as a freshwater strain (as indicated on the culture collection website), served as the reference strain and was grown in the ASN-III medium. All strains showed hampered growth due to change in cultivation medium; nevertheless, they grew and attained reasonable biomass at 8 days of cultivation (Figure 5). Upon GC-MS/MS analysis it was found that the freshwater strains predominantly produced heptadecane while the 

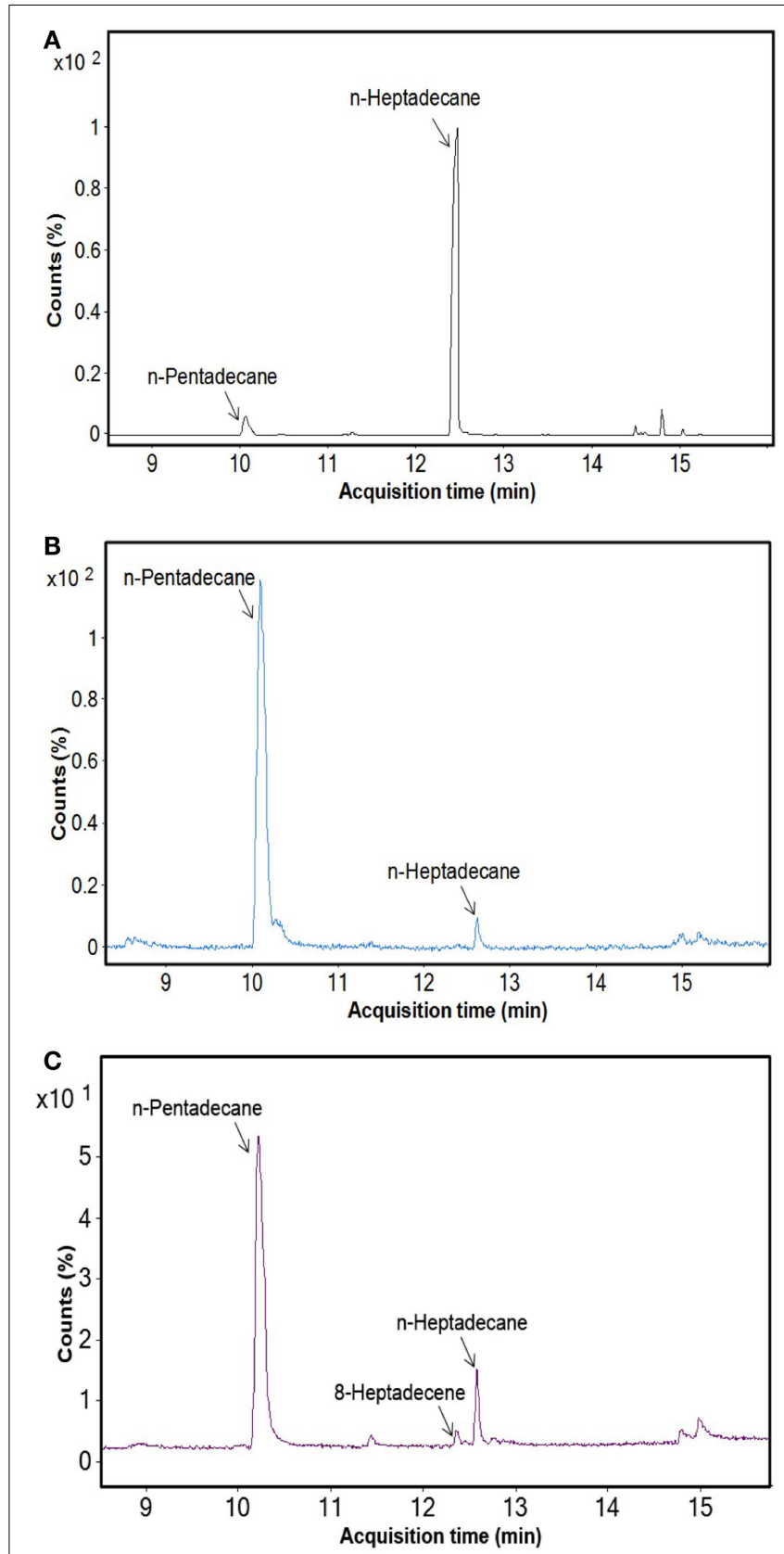

FIGURE 2 | GC-MS profile of freshwater and marine cyanobacteria. (A) Oscillatoria CCC305 strain (freshwater), (B) Oscillatoria formosa BDU3063 (marine), (C) Synechococcus elongatus PCC7942 (positive control).

marine strains produced pentadecane, akin to the trend observed with standard cultivation medium (Figure 5). These findings were a clear indication that a change in the media composition from low- to high-salt concentration or vice versa had no major impact on the chain length of the alkanes produced.

\section{FATTY ACID DISTRIBUTION IN CYANOBACTERIA}

Since environmental factor such as media composition did not contribute to differences in the hydrocarbon chain length in
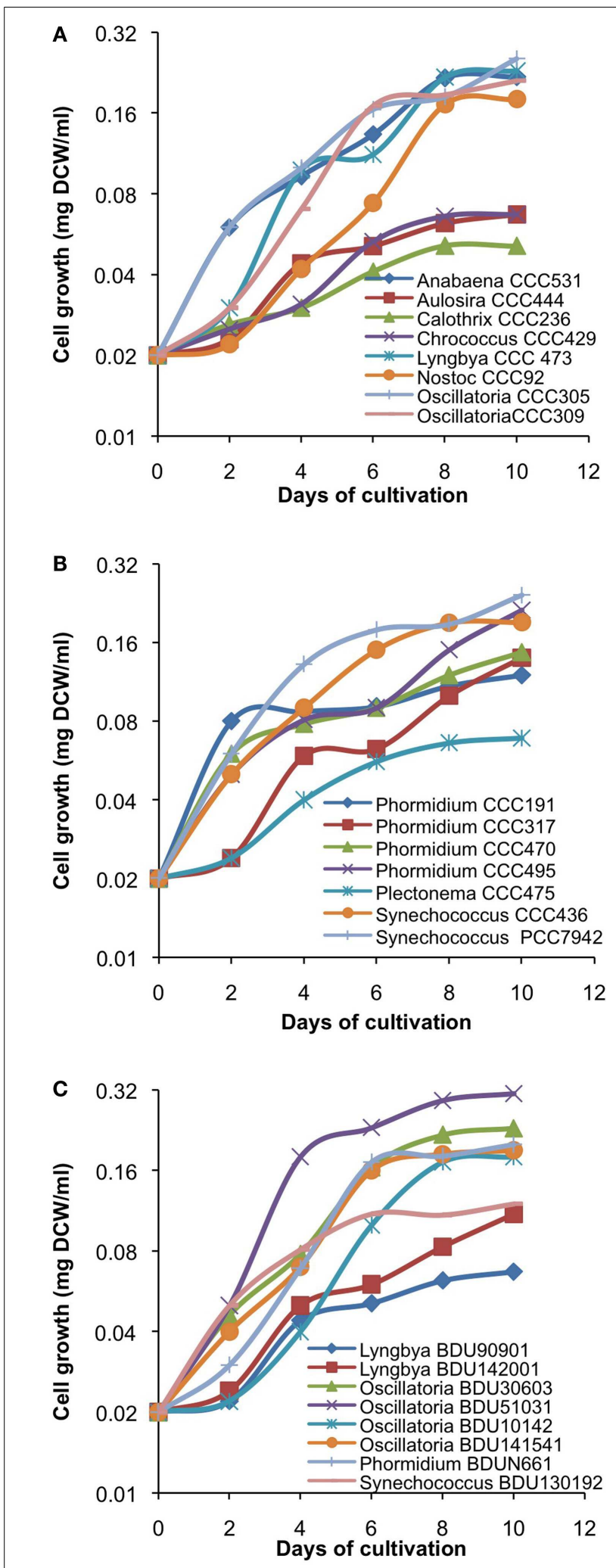

FIGURE 3 | Growth kinetics of freshwater [(A)-from alphabet A-O] and [(B)-from alphabet $P-S$ ] and marine (C) cyanobacterial strains. Data represent average of three independent experiments. 

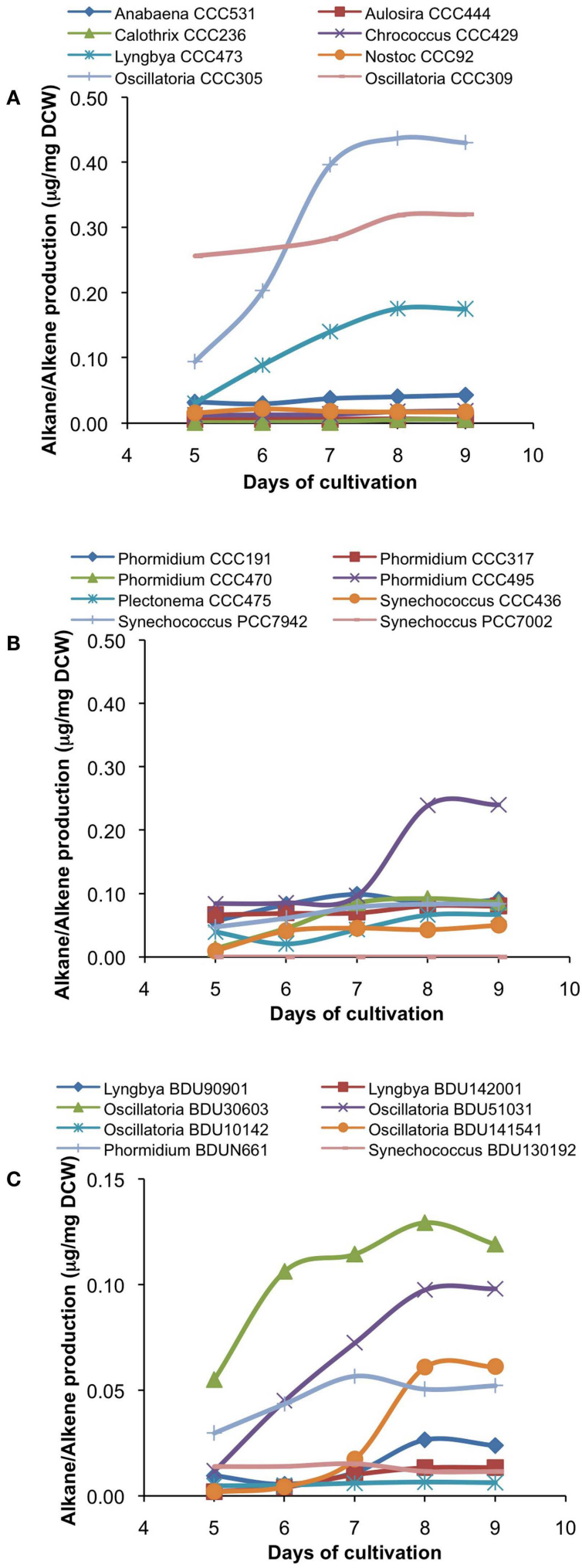

FIGURE 4 | Alkane/alkene production profile of freshwater [(A)-from alphabet A-O] and [(B)-from alphabet P-S] and marine (C) cyanobacterial strains at different days of growth. Data represent average of three independent experiments. freshwater and marine cyanobacteria, we looked at the possible involvement of fatty acid synthesis pathway in determining the hydrocarbon chain length characteristics. We extracted fatty acids from the major alkane-producing cyanobacterial strains, transesterified them and analyzed on GC-MS/MS, as mentioned in Section "Materials and Methods."We found that the marine strains consistently produced higher amount of $\mathrm{C} 16$ chain length fatty acid (Figure 6), which is a precursor for the $\mathrm{C} 15$ chain length alkane (pentadecane) (Schirmer et al., 2010), a characteristics for marine strains. On the other hand, the relative proportion of C18 chain length fatty acids increased significantly in freshwater strains as compared to marine, thus explaining the presence of the higher $\mathrm{C} 17$ chain length (heptadecane) in these strains. However, Phormidium CCC495 produced a much higher quantity of C16 chain length fatty acids though the higher $\mathrm{C} 17$ chain length alkane (heptadecane) was still present (Figure 2A). The positive control S. elongatus PCC7942 strain continued to show a higher level of C16 in concordance with higher production of pentadecane. An intriguing observation was made with regards to the cellular fatty acid composition of both freshwater and marine cyanobacteria. We found that unsaturated fatty acid contents were significantly higher (Figure S3 in Supplementary Material), which were not reflected in the low abundance of alkenes.

\section{PHYLOGENETIC ANALYSIS OF ALKANE-PRODUCING STRAINS}

We selected the alkane-producing cyanobacterial strains and analyzed their phylogenetic properties by aligning the $16 \mathrm{~S} \mathrm{rDNA}$ sequence via Neighbor-Joining method. We found that all marine strains occupied one sub-branch while all freshwater strains occupied another sub-branch (Figure 7A). The branching pattern concurred quite well with alkane chain length, where the pentadecane was associated with the marine sub-branch while heptadecane was associated with the freshwater sub-branch. For this analysis, the $16 \mathrm{~S}$ rDNA sequences of five cyanobacterial strains reported to be hydrocarbon producers by Schirmer et al. (2010) were included: Nostoc PCC73102, S. elongatus PCC6803, S. elongatus PCC7942, Prochlorococcus CCMP1986, and Cyanothece ATCC51142. The information available at the culture collection website indicated Nostoc PCC73102, S. elongatus PCC6803, S. elongatus PCC7942 as freshwater strains, and Prochlorococcus CCMP1986 and Cyanothece ATCC51142 as marine strains. We noted that S. elongatus PCC7942 aligned along with pentadecane-producing strains that originated from the marine habitat (Figure 7B). S. elongatus PCC6803 also clustered with pentadecane producers, though it was reported to be a heptadecane producer (Schirmer et al., 2010). Others aligned in the clusters as expected from their habitat and carbon chain length characteristics.

\section{DISCUSSION}

Recent characterization of the alkane-producing AAR/ADO pathway in cyanobacteria opens up the possibility of producing alkanes within a commercial context using various synthetic biology tools. Cyanobacteria occupying different niches will quite likely have the ability to produce different kinds of hydrocarbon. Prominent among different niches are freshwater and marine habitats. Cyanobacteria from both these habitats have been reported to produce both alkanes and alkenes. However, a detailed study on the 

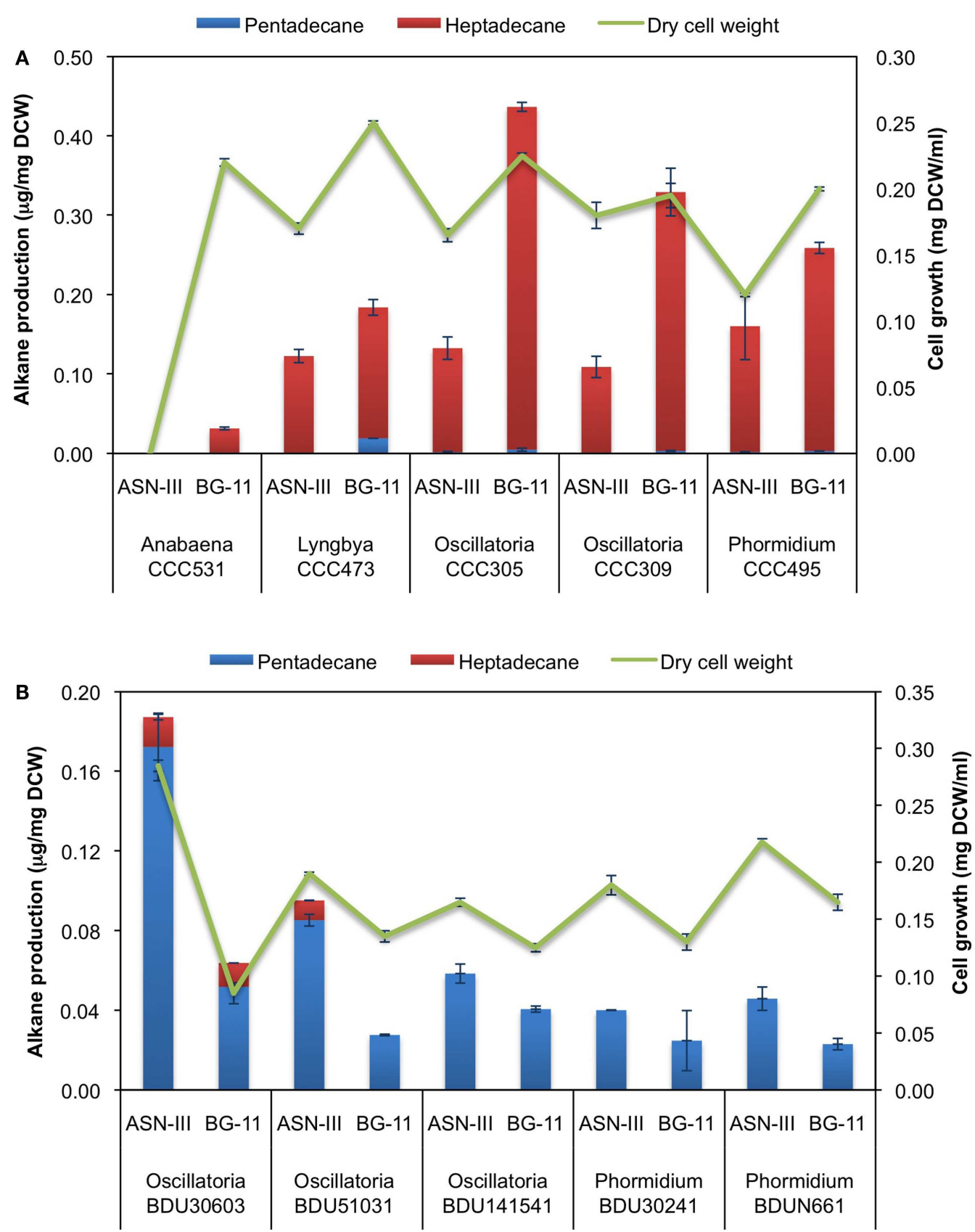

FIGURE 5 | Impact of growth medium on hydrocarbon profile of (A) freshwater and (B) marine cyanobacterial strains. Data represent average and SD of three independent experiments.

characteristics of the alkanes and alkenes produced by the marine and freshwater cyanobacteria is not available.

Given the richness of microbial diversity in India, we obtained freshwater and marine cyanobacterial strains from two Indian culture collections and studied their alkane-producing capacities (Satyanarayana and Johri, 2005). Among the 50 strains, 32 (14 freshwater and 18 marine) strains produced alkanes and to a lesser extent alkenes. The freshwater strains predominantly produced heptadecane while marine strains predominantly produced pentadecane. This unique observation has never been reported before. Furthermore, the lack of unsaturation at the $\alpha$ carbon position would suggest that the AAR and ADO pathway rather than the PKS-type pathway serves as the main route toward alkane/alkene production, corroborating a previous report that a large number of cyanobacteria possess AAR/ADO pathway (Coates et al., 2014).

It is not really known what triggers alkane production in cyanobacteria. Some studies have indicated that environmental 


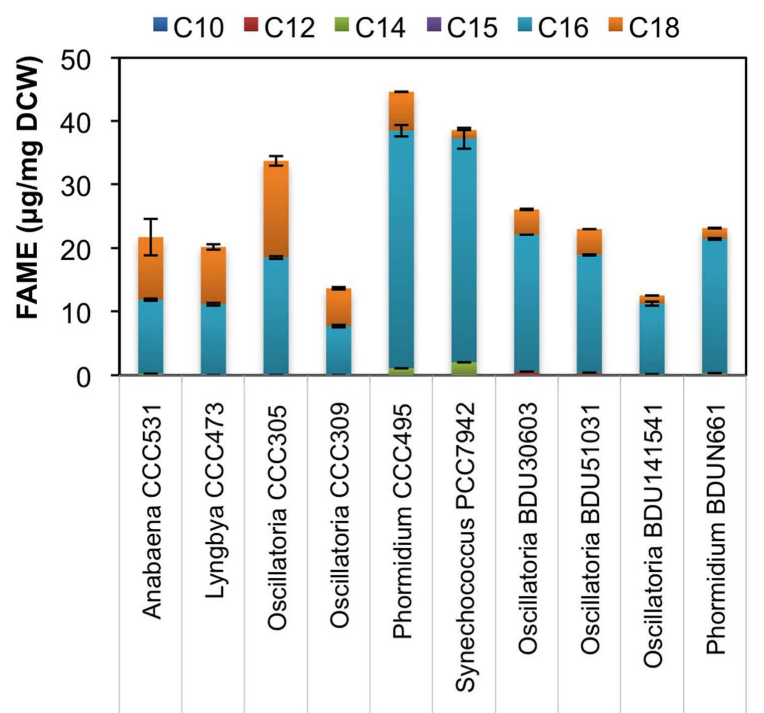

Fresh water strains

Marine strains

FIGURE 6 | Fatty acid chain length profile of freshwater and marine cyanobacterial strains. Cellular fatty acids were extracted from cyanobacteria, trans-esterified to fatty acyl methyl ester (FAME) and analyzed on GC-MS/MS. Data represent average and SD of three independent experiments.

signals might be responsible but no clear conclusion has been drawn (Klähn et al., 2014). It has previously been suggested that media composition, especially salt concentration, may play a role in conferring different alkane chain lengths in freshwater and marine strains. In our case, altering the media composition for freshwater and marine strains had no major impact on the composition of the alkane profile. We considered the metabolic characteristics of the cyanobacteria as another possible determining factor. Alkane production is thought to proceed primarily through the fatty acid synthesis (FAS) pathway followed by reduction and a carbonyl group removal via AAR and ADO enzymes (Schirmer et al., 2010). It was observed previously that although the S. elongatus PCC6803 produced only heptadecane, the recombinant E. coli carrying AAR and ADO of S. elongatus PCC6803 produced a mixture of pentadecane and 8-heptadecene (Schirmer et al., 2010). Similarly, while S. elongatus PCC7942 has a preference for the production of pentadecane with some heptadecane in the ratio of $\sim 3: 1$ (Figure 1), the engineered E. coli expressing the AAR and ADO of this strain produced mainly pentadecane and 8-heptadecene (Figure S4 in Supplementary Material) (Schirmer et al., 2010). These results indicated that perhaps AAR and ADO are not responsible for the variation in the chain length of the alkanes observed between freshwater and marine strains. We further analyzed the properties of fatty acid synthesis pathway of freshwater and marine strains by analyzing the fatty acid content of the alkane-producing strains. We found that all the marine strains predominantly produced C16 chain length (Figure 6), which provides the rational for them being the predominant C15 chain alkane producer. The relative proportion of C18 chain fatty acid increased in freshwater strains, again providing rational for predominantly $\mathrm{C} 17$ chain alkane production by these strains. Interestingly, both the fatty acid and hydrocarbon chain length of S. elongatus PCC7942 exhibit characteristics similar to that of marine species, though it is considered a freshwater species. Overall, these data strongly suggest that alkane chain length is strongly determined by the indigenous fatty acid synthesis pathway of the cyanobacterial strain. Previous study showed clustering of AAR/ADO genes along with a few genes of the fatty acid synthesis pathway, which may indicate a close association of fatty acid and alkane biosynthesis (Klähn et al., 2014). However, we also observed that several fatty acids in these cyanobacteria were unsaturated though this did not correlate with accumulation of the respective alkenes. The unsaturation events were perhaps introduced at a later stage by the known desaturase enzymes, the prominent one being cyanobacterial DesA that introduces double bond at the delta position of fatty acid bound in membrane glycerolipids to provide cold tolerance (Wada et al., 1990). It seems quite possible that alkane synthesis and hydrocarbon chain unsaturation may form diverging pathways from fatty acid synthesis.

Since it was evident from the above observation that genetic factor determines the hydrocarbon chain length in the cyanobacterial strains, we therefore analyzed the phylogenetic properties of alkane-producing strains through alignment of their $16 \mathrm{~S}$ rDNA sequences. Clustering of pentadecane-producing marine cyanobacteria on one side of the evolutionary branch with heptadecane-producing freshwater strains clustering on another suggests that the alkane chain length perhaps evolved according to the natural habitat in which they lived (Figure 7A). What significance this property holds for the cyanobacteria needs to be analyzed further. We also gathered information from the literature regarding the hydrocarbon chain length, $16 \mathrm{~S}$ rDNA sequence, and the natural habitat of some of the alkane/alkene-producing cyanobacteria reported by Schirmer et al. (2010). Again, S. elongatus PCC7942 clustered along with other pentadecane producers from marine source (Figure 7B), though it is considered a freshwater strain within the literature. S. elongatus PCC6803, which has been reported to be a heptadecane producer from freshwater source, surprisingly clustered well with pentadecane-producing marine strains. It is tempting to hypothesize from the phylogenetic analysis that these strains might have evolved from the marine environment. While PCC7942 still retains its pentadecaneproducing ability, PCC6803 may possibly have switched to heptadecane production. This hypothesis would require further investigation. With a few exceptions, our analysis does suggest that the majority of alkane-producing cyanobacterial strains cluster according to their niche area of habitation and alkane chain length.

In summary, we screened and identified cyanobacterial strains that produced more than fivefold higher alkane than the amount produced by previously reported S. elongatus PCC7942. We found that the freshwater strains and marine strains predominantly produced heptadecane and pentadecane, respectively, which correlated well with their phylogenetic clustering. Most importantly, the distinct alkane chain length between freshwater and marine strains is most probably attributed to the subtle differences in the metabolic characteristics of the fatty acid synthesis pathway. 


\section{A}
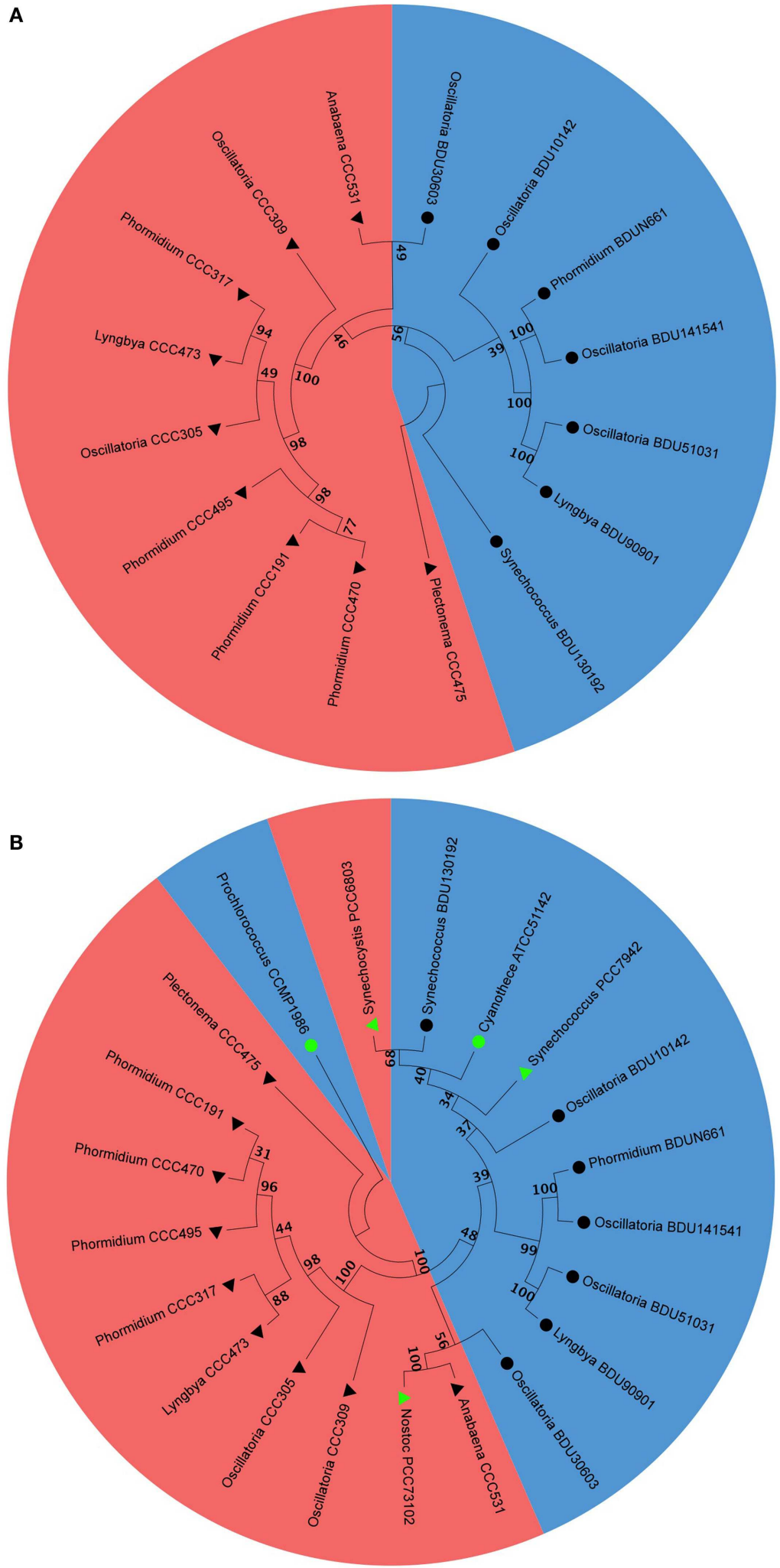

FIGURE 7 | Phylogenetic analysis of marine and freshwater cyanobacteria from Indian culture collection alone (A) and along with some of the published strains (B). Filled triangle represent freshwater, filled circle represent marine, black and green triangles and circles represent cyanobacteria from Indian culture collection and from the published literature, respectively. Blue color and red color shading represent pentadecane and heptadecane producer, respectively. 


\section{AUTHOR CONTRIBUTIONS}

SY designed the study. SY and TF supervised the research. TS performed all the experiments related to cyanobacterial hydrocarbon, fatty acid, and phylogenetic analysis. ZF cloned and expressed hydrocarbon pathway enzymes in E. coli. SY and TS wrote the manuscript.

\section{ACKNOWLEDGMENTS}

We are thankful to IARI and NFMC for providing cyanobacterial strains for this study. We acknowledge financial support from Department of Biotechnology, Govt. of India for carrying out this work.

\section{SUPPLEMENTARY MATERIAL}

The Supplementary Material for this article can be found online at http://journal.frontiersin.org/article/10.3389/fbioe.2015.00034

\section{REFERENCES}

Ahmad, I., Fatma, Z., Yazdani, S. S., and Kumar, S. (2012). DNA barcode and lipid analysis of new marine algae potential for biofuel. Algal Res. 2, 10-15. doi:10.1016/j.algal.2012.10.003

Bloch, J. F., and Tardieu-Guigues, E. (2014). Marine biotechnologies and synthetic biology, new issues for a fair and equitable profit-sharing commercial use. Mar. Genomics 17, 79-83. doi:10.1016/j.margen.2014.07.003

Cameron, R. E. (1962). Species of Nostoc Vaucher occurring in the Sonoran desert in Arizona. Trans. Am. Microsc. Soc. 81, 379-384. doi:10.2307/3223790

Coates, R. C., Podell, S., Korobeynikov, A., Lapidus, A., Pevzner, P., Sherman, D. H., et al. (2014). Characterization of cyanobacterial hydrocarbon composition and distribution of biosynthetic pathways. PLoS One 9:e85140. doi:10.1371/journal. pone. 0085140

Felsenstein, J. (1985). Confidence limits on phylogenies: an approach using the bootstrap. Evolution 39, 783-791. doi:10.2307/2408678

Garcia-Pichel, F., López-Cortés, A., and Nübel, U. (2001). Phylogenetic and morphological diversity of cyanobacteria in soil desert crusts from the Colorado plateau. Appl. Environ. Microbiol.67, 1902-1910. doi:10.1128/AEM.67.4.1902-1910.2001

Hernandez-Prieto, M. A., and Futschik, M. E. (2012). CyanoEXpress: a web database for exploration and visualization of the integrated transcriptome of cyanobacterium Synechocystis sp. PCC6803. Bioinformation 8, 634-638. doi:10.6026/ 97320630008634

Klähn, S., Baumgartner, D., Pfreundt, U., Voigt, K., Schön, V., Steglich, C., et al. (2014). Alkane biosynthesis genes in cyanobacteria and their transcriptional organization. Front. Bioeng. Biotechnol. 2:24. doi:10.3389/fbioe.2014.00024

Li, N., Chang, W. C., Warui, D. M., Booker, S. J., Krebs, C., and Bollinger, J. M. Jr. (2012). Evidence for only oxygenative cleavage of aldehydes to alk (a/e)nes and formate by cyanobacterial aldehyde decarbonylases. Biochemistry 51, 7908-7916. doi:10.1021/bi300912n

Li, N., Nørgaard, H., Warui, D. M., Booker, S. J., Krebs, C., and Bollinger, J. M. Jr. (2011). Conversion of fatty aldehydes to alka(e)nes and formate by a cyanobacterial aldehyde decarbonylase: cryptic redox by an unusual dimetal oxygenase. J. Am. Chem. Soc. 133, 6158-6161. doi:10.1021/ja2013517

Martins, J., Peixe, L., and Vasconcelos, V. M. (2011). Unraveling cyanobacteria ecology in wastewater treatment plants (WWTP). Microb. Ecol. 62, 241-256. doi:10.1007/s00248-011-9806-y

Mendez-Perez, D., Begemann, M. B., and Pfleger, B. F. (2011). Modular synthaseencoding gene involved in $\alpha$-olefin biosynthesis in Synechococcus sp. strain PCC 7002. Appl. Environ. Microbiol. 77, 4264-4267. doi:10.1128/AEM.00467-11

Miller, S. R., and Castenholz, R. W. (2000). Evolution of thermo tolerance in hot spring cyanobacteria of the genus Synechococcus. Appl. Environ. Microbiol. 66, 4222-4229. doi:10.1128/AEM.66.10.4222-4229.2000
Nadeau, T. L., and Castenholz, R. W. (2000). Characterization of psychrophilic oscillatorians (cyanobacteria) from Antarctic melt water ponds. J. Phycol. 36, 914-923. doi:10.1046/j.1529-8817.2000.99201.x

Nei, M., and Kumar, S. (2000). Molecular Evolution and Phylogenetics. New York, NY: Oxford University Press.

Reed, R. H., Chudek, J. A., Foster, R., and Stewart, W. D. P. (1984). Osmotic adjustment in cyanobacteria from hypersaline environments. Arch. Microbiol. 138, 333-337. doi:10.1007/BF00410900

Rippka, R., Deruelles, J., Waterbury, J. B., Herdman, M., and Stanier, R. Y. (1979). Generic assignments, strain histories and properties of pure cultures of cyanobacteria. J. Gen. Microbiol. 111, 1-61. doi:10.1099/00221287-111-1-1

Saitou, N., and Nei, M. (1987). The neighbor-joining method: a new method for reconstructing phylogenetic trees. Mol. Biol. Evol. 4, 406-425.

Sarchizian, I., and Ardelean, I. I. (2010). Axenic culture of a diazotrophic filamentous cyanobacterium isolated from mesothermal sulphurous springs (Obanul mare - Mangalia). Rom. J. Biol. Plant Biol. 55, 47-53.

Satyanarayana, T., and Johri, B. N. (2005). Microbial Diversity: Current Perspectives and Potential Applications. New Delhi: IK International Pvt Ltd.

Schirmer, A., Rude, M. A., Li, X., Popova, E., and del Cardayre, S. B. (2010). Microbial biosynthesis of alkanes. Science 329, 559-562. doi:10.1126/science. 1187936

Speight, J. G. (ed.) (1997). Petroleum Chemistry and Refining. Laramie: CRC Press, 9-26.

Tamura, K., Stecher, G., Peterson, D., Filipski, A., and Kumar, S. (2013). MEGA6: molecular evolutionary genetics analysis version 6.0. Mol. Biol. Evol. 30, 2725-2729. doi:10.1093/molbev/mst197

Timilsina, G. R. (2013). Biofuels in the long-run global energy supply mix for transportation. Philos. Trans. A Math. Phys. Eng. Sci. 372, 20120323. doi:10.1098/rsta. 2012.0323

Wada, H., Gombos, Z., and Murata, N. (1990). Enhancement of chilling tolerance of a cyanobacterium by genetic manipulation of fatty acid desaturation. Nature 347, 200-203. doi:10.1038/347200a0

Winters, K., Parker, P. L., and Baalen, C. V. (1969). Hydrocarbons of blue-green algae: geochemical significance. Science 163, 467-468. doi:10.1126/science.163. 3866.467

Zhang, J., Lu, X., and Li, J.-J. (2013). Conversion of fatty aldehydes into alk(a/e)nes by in vitro reconstituted cyanobacterial aldehyde-deformylating oxygenase with the cognate electron transfer system. Biotechnol. Biofuels 6, 86. doi:10.1186/17546834-6-86

Zhang, X.-C., Millet, Y., Ausubel, F. M., and Borowsky, M. (2014). Nextgen sequencing-based mapping and identification of ethyl methanesulfonateinduced mutations in Arabidopsis thaliana. Curr. Protoc. Mol. Biol. 108, 7.18.1-7.18.16. doi:10.1002/0471142727.mb0718s108

Conflict of Interest Statement: The authors declare that the research was conducted in the absence of any commercial or financial relationships that could be construed as a potential conflict of interest.

Received: 02 December 2014; accepted: 02 March 2015; published online: 16 March 2015.

Citation: Shakeel T, Fatma Z, Fatma T and Yazdani SS (2015) Heterogeneity of alkane chain length in freshwater and marine cyanobacteria. Front. Bioeng. Biotechnol. 3:34. doi: 10.3389/fbioe.2015.00034

This article was submitted to Synthetic Biology, a section of the journal Frontiers in Bioengineering and Biotechnology.

Copyright (c) 2015 Shakeel, Fatma, Fatma and Yazdani. This is an open-access article distributed under the terms of the Creative Commons Attribution License (CC BY). The use, distribution or reproduction in other forums is permitted, provided the original author(s) or licensor are credited and that the original publication in this journal is cited, in accordance with accepted academic practice. No use, distribution or reproduction is permitted which does not comply with these terms. 